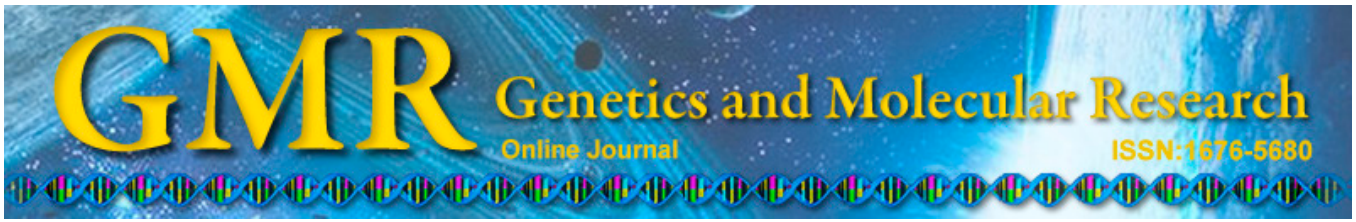

\title{
Characterization and expression analysis of the prophenoloxidase activating factor from the mud crab Scylla paramamosain
}

J. Wang ${ }^{1,2}$, K.J. Jiang ${ }^{1}$, F.Y. Zhang ${ }^{1}$, W. Song ${ }^{1}$, M. Zhao ${ }^{1,2}$, H.Q. Wei ${ }^{1,2}$, Y.Y. Meng ${ }^{1,2}$ and L.B. Ma ${ }^{1}$

${ }^{1}$ East China Sea Fisheries Research Institute, Chinese Academy of Fishery Sciences, Shanghai, China

${ }^{2}$ College of Fisheries and Life Science, Shanghai Ocean University, Shanghai, China

Corresponding author: L.B. Ma

E-mail address: malingbo@vip.sina.com

Genet. Mol. Res. 14 (3): 8847-8860 (2015)

Received November 25, 2014

Accepted April 27, 2015

Published August 3, 2015

DOI http://dx.doi.org/10.4238/2015.August.3.8

ABSTRACT. Prophenoloxidase activating factors (PPAFs) are a group of clip domain serine proteinases that can convert prophenoloxidase (pro-PO) to the active form of phenoloxidase (PO), causing melanization of pathogens. Here, two full-length PPAF cDNAs from Scylla paramamosain (SpPPAF1 and SpPPAF2) were cloned and characterized. The full-length $S p P P A F 1$ cDNA was 1677 bp in length, including a 5'-untranslated region (UTR) of $52 \mathrm{bp}$, an open reading frame (ORF) of $1131 \mathrm{bp}$ coding for a polypeptide of 376 amino acids, and a 3'-UTR of $494 \mathrm{bp}$. The full-length SpPPAF2 cDNA was 1808 bp in length, including a 5'-UTR of $88 \mathrm{bp}$, an ORF of $1125 \mathrm{bp}$ coding for a polypeptide of 374 amino acids, and a 3'-UTR of $595 \mathrm{bp}$. The estimated molecular weight of $S P P P A F 1$ and $S p P P A F 2$ was 38.43 and $38.56 \mathrm{kDa}$ with an isoelectric point of 7.54 and 7.14, respectively. Both $S p P P A F 1$ and $S p P P A F 2$ proteins consisted of a signal peptide, a characteristic structure of clip domain, and a carboxyl-terminal trypsin- 
like serine protease domain. Expression analysis by qRT-PCR showed that $S p P P A F 1$ mRNA was mainly expressed in the gill, testis, and hemocytes, and SpPPAF2 mRNA was mainly expressed in hemocytes. In addition, $S p P P A F 1$ and $S p P P A F 2$ mRNA was expressed in a timedependent manner after Vibrio parahaemolyticus challenge. The results showed that expression of both SpPPAF1 and SpPPAF2 was related to the bacterial challenge but the expression patterns differed. These findings suggest that $S P P P A F$ is a serine proteinase and may be involved in the pro-PO activation pathway of the crab innate immune system.

Key words: Scylla paramamosain; Quantitative real-time PCR; Prophenoloxidase activating factor

\section{INTRODUCTION}

As invertebrates lack an adaptive immune system, melanin formation becomes one of their major innate immune responses (Ma et al., 2014). Melanization participates in various physiological processes, including antimicrobial immune responses (Wang et al., 2014). The process of melanization is controlled by the prophenoloxidase (pro-PO) activating system, which plays an important role in cuticular sclerotization and in defense against pathogens and parasites in invertebrates (Söderhäll and Cerenius, 1998). Usually, this system is activated by pattern-recognition proteins bound to $\beta-1,3$-glucans, lipopolysaccharides, peptidoglycans (i.e., $\beta G B P$, LGBP, and PGBP) or by other compounds, such as endogenous factors produced upon tissue damage (Cerenius and Söderhäll, 2004). A cascade of serine proteinases will then catalyze the proteolysis of the pro-form of the prophenoloxidase activating factors (pro-PPAFs) into active PPAFs. These PPAFs then convert pro-PO to the active form of PO, causing melanization of pathogens (Söderhäll et al., 1994; Cerenius and Söderhäll, 2004). Actually, the activated PO can catalyze the enzymatic conversion of monophenolic and diphenolic substances to quinones, which undergo further reactions to produce cytotoxic intermediates and melanin that ultimately encapsulates and demolishes certain pathogens (Amparyup et al., 2013). The presence of specific proteinase inhibitors in this system prevents superfluous activation, such as the generation of factors with anti-microbial, cytotoxic, opsonic, or encapsulation promoting activities (Cerenius and Söderhäll, 2004).

The pro-PO activating system consists of a cascade of enzymatic reactions controlled by serine proteinases (SPs) and their homologues (SPHs). A common feature of these enzymes is that they contain at least one disulfide-bridged structure known as a clip domain, and therefore they are also called clip domain serine proteinases (clip-SPs) (Jiang and Kanost, 2000). SPHs are similar in amino acid sequence to SPs but apparently lack amidase activity since one or more of the catalytic residues are missing or mutated (Jiménez-Vega et al., 2005). SPs and SPHs have become a hotspot of recent research and were found to have diverse functions, such as melanocyte encapsulation, induction of anti-microbial peptide synthesis, embryo protection, and cytokine activation (Kawabata et al., 1996; Jiang and Kanost, 2000; Kanost et al., 2001). To elucidate the pro-PO activating system of crustaceans at the molecular level, it is essential to purify and characterize SPs and SPHs involved in this system.

One of the major steps in the pro-PO activating system is the activation of serine proteinases of the pro-PO pathway by PPAFs. PPAFs also belong to the SPs/SPHs family and can 
convert pro-PO to the active form of PO, causing melanization of pathogens. Several PPAFs have been reported in crustaceans, including the blue crab Callinectes sapidus (Buda and Shafer, 2005), the freshwater crayfish Pacifastacus leniusculus (Wang et al., 2001), the black tiger shrimp Penaeus monodon (Charoensapsri et al., 2009, 2011; Ma et al., 2014), and the white shrimp Litopenaeus vannamei (Jang et al., 2011). According to some previous studies, PPAFs have different functions in different species. For example, PPAF in blue crabs can control and regulate molting (Buda and Shafer, 2005); PPAF II in Holotrichia diomphalia serves as a cofactor of PPAF-I and is involved in the activation of the pro-PO system (Kwon et al., 2000); in silkworm Bombyx mori, the purified PPAF enzyme itself can cleave prophenoloxidase at Arg 51 and fully activate the proenzyme (Satoh et al., 1999). Several PPAF protein sequences have been purified and cloned from some arthropod species, but few from crustacean species. In addition, how these PPAFs activate the pro-PO activating system has not been elucidated at the molecular level. Given the importance of PPAFs in the pro-PO activating system and in the immune defense mechanism of cultivated Scylla paramamosain, the present study intended to characterize two full-length cDNAs of PPAF in $S$. paramamosain (SpPPAF1 and SpPPAF2), and detect the relative expression profiles in different tissues and the temporal expression pattern of SPPPAF mRNA in the megalopa stage after bacterial challenge, attempting to clarify their potential roles in immune responses in S. paramamosain.

\section{MATERIAL AND METHODS}

\section{Material and reagents}

Healthy mud crabs weighing $200 \pm 20$ g were collected from Hainan Island, China. Different organs and tissues, including the hepatopancreas, muscle, gill, testis, heart, ovary, and hemocytes were immediately collected and preserved in liquid nitrogen for RNA extraction. Hemocytes were withdrawn with a needle from the unsclerotized membranes of the crab into a syringe containing the pre-cooled $\left(-4^{\circ} \mathrm{C}\right)$ anticoagulant $(0.45 \mathrm{M} \mathrm{NaCl}, 0.1 \mathrm{M}$ glucose, 30 $\mathrm{mM}$ trisodium citrate, $26 \mathrm{mM}$ citric acid, and $10 \mathrm{mM}$ EDTA, $\mathrm{pH}$ 4.6) to reach a volume ratio of $1: 1$, and then pelleted by centrifugation $(8000 \mathrm{rpm})$ (Allegra ${ }^{\mathrm{TM}}$ 64R Centrifuge, Beckman Coulter, USA) at $4^{\circ} \mathrm{C}$ for $10 \mathrm{~min}$.

\section{cDNA library construction}

An $S$. paramamosain cDNA library was constructed successfully using a SMART ${ }^{\mathrm{TM}}$ cDNA library construction kit (Clontech, Palo Alto, CA, USA) and all the EST sequences were subjected to BLAST analysis (Zhang et al., 2011). BLAST analysis of all the EST sequences showed that two EST sequences were highly similar to the previously identified PPAFs, and therefore they were selected for further cloning of SpPPAF cDNA.

\section{Obtaining the full-length cDNA of the SpPPAF1 and SpPPAF2 genes}

Total RNA was isolated from the hemocytes using Unizol reagents (Biostar, Shanghai, China) following the manufacturer protocol, and then stored at $-80^{\circ} \mathrm{C}$. The quality and concentration were checked by agarose gel electrophoresis and spectrophotometry (DU 800, Beckman Coulter, USA). First-strand cDNA synthesis was performed using M-MLV reverse 
transcriptase (Promega Corporation, Madison, WI, USA) to transcribe poly(A) mRNA with Oligo-dT and random 6-mer primers. The reaction conditions were as recommended by the manufacturer. The missing 5' and 3' ends of the PPAF cDNA of S. paramamosain were obtained using the rapid amplification of cDNA ends (RACE) method, which was performed using the SMART ${ }^{\mathrm{TM}}$ RACE cDNA amplification kit (Clontech, Palo Alto, CA, USA) according to the manufacturer protocol. The products were checked by agarose gel and purified using the Agarose Gel DNA Purification kit version 2.0 (TaKaRa, Dalian, China), and then cloned into the pMD19-T vector (TaKaRa, Dalian, China) and sequenced. Primers for 5'- and 3'-RACE are provided in Table 1.

Table 1. Primers used to sequence prophenoloxidase activating factor cDNA from Scylla paramamosain (SpPPAF).

\begin{tabular}{ll}
\hline Primer name & Sequence $\left(5^{\prime}-3^{\prime}\right)$ \\
\hline SpPPAF1-3GSP & ATTCGTGGTGGGTATCACAAGC \\
SpPPAF1-5GSP & CACCACCAGCACCATCATCACC \\
SpPPAF2-3GSP & CAACAGGGCTGAAACAAGAAGGT \\
SpPPAF2-5GSP & TCACAAACTCCAACTGGTGGTCAT \\
18SRNA-RT-F & GGGGTTTGCAATTGTCTCCC \\
18SRNA-RT-R & GGTGTGTACAAAGGGCAGGG \\
SpPPAF1-RT-F & GTTTGCTTATGCTGCTGGTGG \\
SpPPAF1-RT-R & TAATGGGGAGGAGGACTTGCTG \\
SpPPAF2-RT-F & CCAGGACATCACCACGGAGCAG \\
SpPPAF2-RT-R & CAGATGGGCACCAAGTGAACAGG \\
\hline
\end{tabular}

\section{Sequence analysis}

Identity searches for the nucleotide and protein sequences were performed using the BLAST algorithm at NCBI (http://www.ncbi.nlm.nih.gov/). The deduced amino acid sequence was analyzed with the Expert Protein Analysis System (http://www.expasy.org/). Amino acid sequences from various species were retrieved from NCBI GenBank and analyzed using the Vector NTI Suite 11.5, Clustal W Multiple Alignment program (http://www.ebi.ac.uk/clustalw/). A neighbor-joining (NJ) phylogenetic tree was constructed using the MEGA software version 4.1 (Tamura et al., 2011) and the confidence level in the tree was obtained using 1000 bootstraps. The secondary structure of SPPPAF was predicted by the application of the hierarchical neural network (http://www.expasy.org/). The 3-D structure of SpPPAF was simulated using the SWISS-MODEL long-distance server (http://www.expasy.org/).

\section{qRT-PCR analysis of $S p P P A F$ in different tissues}

Total RNA $(1 \mu \mathrm{g})$ was reverse transcribed with the ReverTraAce qPCR RT kit (Toyobo) for first strand cDNA synthesis. The products were diluted 10 -fold and stored at $-20^{\circ} \mathrm{C}$ until quantitative real-time PCR analysis (qRT-PCR). The transcript expression profiles of $S p P P A F$ were detected using qRT-PCR. A pair of $18 S$ rRNA primers designed from the $18 \mathrm{~S}$ rRNA sequence from the NCBI GenBank (GenBank accession No. FJ646616.1) were used as the internal control. Expression levels were calculated using the standard curve method. The SYBR Green qRT-PCR assay using Power SYBR Green PCR master mix (Applied Biosystems, Foster City, CA, USA) was carried out in an ABI StepOnePlus Detection system (Applied Biosystems). Amplifications were performed on a $96-$ well plate with a $20-\mu \mathrm{L}$ reaction 
volume containing $10 \mu \mathrm{L}$ SYBR Premix Taq TM, $0.8 \mu \mathrm{L} 10 \mathrm{mM}$ PCR forward primer, $0.8 \mu \mathrm{L}$ $10 \mathrm{mM}$ PCR reverse primer, $0.4 \mu \mathrm{L}$ ROX Reference DyeI, $2.0 \mu \mathrm{L}$ cDNA template, and $6.0 \mu \mathrm{L}$ diethylpyrocarbonate water (DEPC-water). The reaction conditions for the SYBR Green qRTPCR were $10 \mathrm{~min}$ at $94^{\circ} \mathrm{C}$, followed by 45 cycles of $94^{\circ} \mathrm{C}$ for $15 \mathrm{~s}$ and $60^{\circ} \mathrm{C}$ for $1 \mathrm{~min}$, and extension at $72^{\circ} \mathrm{C}$ for $10 \mathrm{~min}$. Primers used in the qRT-PCR are provided in Table 1.

\section{Temporal expression pattern of SpPPAF mRNA in megalopa after bacterial chal- lenge}

Quantitative RT-PCR was used to analyze the expression level of SpPPAF mRNA in the megalopa stage of $S$. paramamosain after challenge with Vibrio parahaemolyticus. The bacterial challenge experiment was performed by adding $2.3 \times 10^{6} \mathrm{CFU} / \mathrm{mL}$ of $V$. parahaemolyticus suspended in physiological saline into the plastic tanks used to culture the larvae. Untreated larvae were used as the control group. Larvae at the megalopa stage were randomly placed into two tanks, and from each tank five individuals were collected at $0,3,6,9,12,24$, 48, and $72 \mathrm{~h}$. Three replicates were employed for each sampling time point. RNA isolation, cDNA synthesis, and qRT-PCR analysis were carried out as described above. The control group was used as the calibrator.

\section{RESULTS}

\section{Cloning of $\operatorname{SpPPAF}$}

Two full-length cDNA sequences of PPAF from S. paramamosain (SpPPAF1 and $S p P P A F 2$ ) were obtained in this study (GenBank accession No. KP143941-KP143942). The full-length $S p P P A F 1$ cDNA was $1677 \mathrm{bp}$, including a 5'-untranslated region (UTR) of $52 \mathrm{bp}$, an open reading frame (ORF) of $1131 \mathrm{bp}$ coding for a polypeptide of 376 amino acids, and a 3'-UTR of $494 \mathrm{bp}$ (Figure 1a). The full-length SpPPAF2 cDNA was $1808 \mathrm{bp}$, including a 5'UTR of $88 \mathrm{bp}$, an ORF of $1125 \mathrm{bp}$ coding for a polypeptide of 374 amino acids, and a 3'-UTR of $595 \mathrm{bp}$ (Figure 1b). Both sequences had a potential polyadenylation sequence (AATAAA) and a poly(A) tail.

As predicted by the SignalP software (http://www.cbs.dtu.dk/services/SignalP), residues 1 to 22 that represent the putative signal peptide sequence were found in both SpPPAF1 and $S p P P A F 2$. Therefore, mature SpPPAF1 was composed of 354 amino acid residues with a theoretical isoelectric point (pI) and predicted molecular weight of 7.54 and $38.43 \mathrm{kDa}$, respectively, and the mature SPPPAF 2 was composed of 352 amino acid residues with a theoretical $\mathrm{pI}$ and predicted molecular weight of 7.14 and $38.56 \mathrm{kDa}$, respectively.

\section{Sequence analysis}

An N-terminal clipdomain and a C-terminaltrypsin-like serine proteinase domain were found in the deduced amino acid sequences of both SPPPAF1 and SpPPAF2. The essential six conserved cysteine residues in the clip domain, which form the three disulfide bonds and the three conserved serine residues of a typical catalytic triad (i.e., His, Asp, and Ser) of a typical trypsin-like SP domain, were found in the SPPPAF sequences. 
a

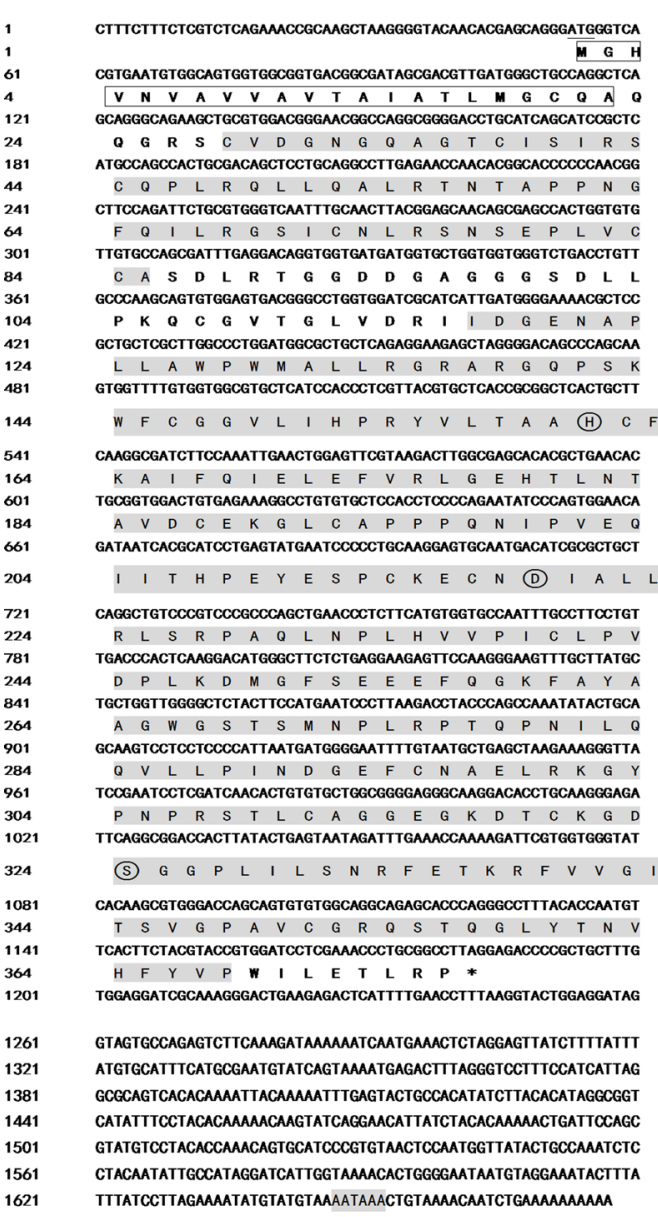

b

1 61
1 12 12
GTTCAGAGGCAGGaGtGAGTTGGTGTAGGACACAGCTTTCTCTGCTECTGCGAGACCCGA GGGATTGAGAAACGAGACTTGAGTAAGAATGGGTCACGTGAATCCGGCGGTGGTGGCGGT M G H V P P V V A V AGCGGCTCTGGCGACACTGATGAGCTGTCAGGCTCAGGAGGGCCGAAGCTGCGTGGACGG O

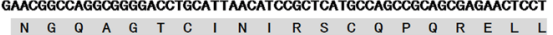

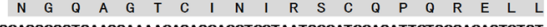
GCAGGCGGTGAAGGAAAACAGAGCACCTOCTAATGCCATCCAGATTCTGCGACAGTCTGT Q A V K E N R A P P N A I O 1 L R Q S V V GTGTAAGTCCTGAGCAGGGAAAGACTACTGGTGTGCTGCGCCCAGAGTAGAAGCACAGG


GACAACTACAAGTGCAGGTAGGGGTAGCGGGGGTGACCTGTTGCCCAGAAATTGCGGGAG

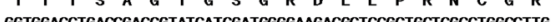

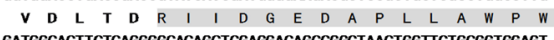
GATGGCACTTCTCAGGGGCAGAGCTCGAGGACAGCGGGCTAACTGGTTCTGGGGTGGAGT

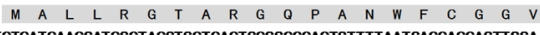
TCTCATCAACGATCGCTACGTGCTCAGTGCGGCCCACTGTTTTAATEACCACCAGTTGGA L I N $\quad$ D $R$ R $\quad$ Y V V L GTTTGTGAGGCTGGGCGAACACACACTCTCCACAACAGAGGACTGCCAGTCAGGCGTGTG F V R L G E H T L S T T E D C Q Q S TECCCCGCCGCCCCAGGACATCACCACGGAGCAGATCATCATTCACССGCAGTATAMATC $\begin{array}{llllllllllllllllllll}A & P & P & P & Q & D & \text { I } & T & T & E & Q & \text { I } & \text { I } & \text { I } & \text { H } & P & 0 & \text { Y } & K & S\end{array}$ ACСGTGCCGGCAGTGCAACGACATCGCGCTCCTCAGGCTGTCTAGTCCCGTCCAGGTGCA

P C R Q C N (D) I A L L R L S S P V O L H СCCTGTTCACTTGGTGCCCATCTGCGTCOCTGTCGACOCAGTGAAGGACATGGGATTCTC $\begin{array}{lllllllllllllllllllll}P & V & H & L & V & P & \text { I } & C & V & P & V & D & P & V & K & D & M & G & F & S\end{array}$ CGAGGCAGACTTCCAGGGAAGCATGCCTGGGCCGCCGGCTGGGGCTCCATCTCCAGGAG E A D TCCCTTGAGGGTGAGTATCCCTGACAGGCTGCAGCAGGTGTTCCTCCCCATCCGCGAGGA $P L R \quad R \quad S \quad I \quad P \quad D \quad T \quad L \quad Q \quad Q \quad V \quad F \quad L \quad P \quad I \quad R E$ CAGGeTCTGCCCGCTGCTGAAGAGAGGCTACCCCGMOCCCGCATGGTGCTGTGTCCTGG $T V C P L L K R G Y P E P R M V L C A G$

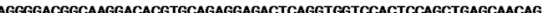

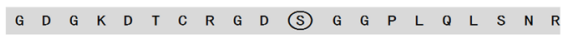
GGCTGAAACAAGAAGGTTTGTGGTTGGCATTACTAGTGTTGGTCCCGAGGTGTGTGGCAG A E I T R R F $F \quad V \quad V \quad G \quad I \quad T$ S AAAGAGCACCCAGGCACTGTACACCAGTGTCCACTTCTACGTGCAGTGGATCCTGGAAM K S T Q A L Y T S V H F Y V Q W I L E N

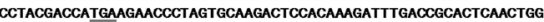
$\mathrm{L} R \mathrm{P} *$

GCCACGTGAGCCITTCACATTATGCGGTTTCTCGTCGCATCTAAATACGGTCCCGGACAC AACAGTCGACTGATAATTTCTATGTCCCTCCTCACACTGTGCGGCTGCTGGTGATTGACC GGTCCGGACCCGGATGCAGTTGCGGCGTOCCACTGGTCTTGTCCGGTCCCGTACCCGTGC GCTGTGGGAGTEGGCTITAAATITAAATAGAAGTCTTCACAGTGCGCGGTTAGTAGCCGC ACAGGCACTGTGTGACAAACATITCTAIAMAGGATTTCAATGTGTGGGGAGCCGGATGA TTGTGCGGCAGCAATCGCAAGCATTTCCGATCCGGCAGCCGGGCCAAGAGGCGGCGAGAA ATCACATAATGTGAAAGAGGCTTCAGATATGGAGGACTCTTACAAGCAGCATCAATCAGG AGTTTTCITTCTCACTCTCTCCAGCGTAAACTGTAGTCATGACAGCACGAGgAaTCAAAT TAAITTCATTCATGAaGACTTATATGGG TTTGIAATAATAAATTTAGITTATGTGCCAAA AAAAAAA

Figure 1. Nucleotide sequence and deduced amino-acid sequence of prophenoloxidase activating factor cDNA from Scylla paramamosain (a) SPPPAF1 and (b) SpPPAF2. The start (ATG) and stop (TAA) codons are underlined and the instability motif (AATAAA) is shaded. The putative sequence of the signal peptide is boxed. The putative $\mathrm{N}$-terminal clipdomain and C-terminaltrypsin-like serine proteinase domain are in gray. Open circles indicate the three conserved serine residues of the typical catalytic triad (His, Asp, and Ser).

According to BLASTP searches (http://blast.ncbi.nlm.nih.gov/Blast.cgi) of the nonredundant protein database at GenBank, the deduced amino-acid sequences of SpPPAF1 and SpPPAF 2 shared the highest identity (78 and $68 \%$, respectively) with the PPAF of $C$. sapidus (GenBank accession No. ABG67960.1). Amino acid sequence alignment was conducted between SpPPAF and other SPs/SPHs reported from other organisms using Vector NTI Suite Advance 11.5 (Figure 2). It was found that some amino acid residues were highly conserved in different species. Interestingly, the predicted amino acid residues representing a clip domain (six cysteine residues) and a SP domain (His, Asp, and Ser) were located at the N- and C-termini, respectively, indicating that these two proteins may belong to the family of clipSPs. In addition, an NJ phylogenetic tree was generated according to the amino acid sequence 
alignment using MEGA 4.1 (Figure 3). Phylogenetic analysis indicated that $S p P P A F$ was more similar to the PPAF of C. sapidus. This result is consistent with the status of systematic evolution, indicating that the PPAF gene could be a molecular index for systematic evolution.

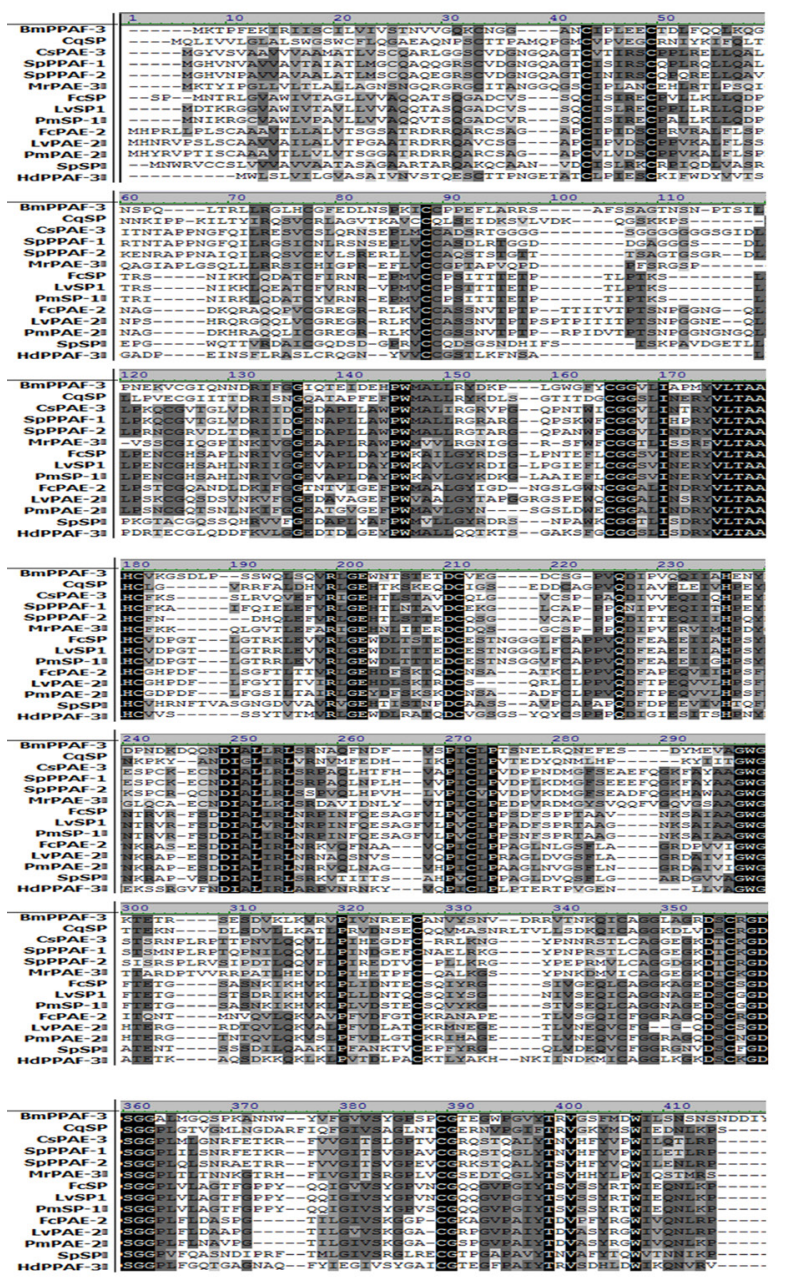

Figure 2. Multiple alignments of the amino acid sequence of Scylla paramamosain prophenoloxidase activating factor $(\mathrm{SpPPAF})$ and other serine proteinases/serine proteinase homologues (SPs/SPHs) from other species. Gaps $(-)$ were introduced to maximize the alignment. The relationships between residues are indicated as follows: nonsimilar residues, black letters on a white background; conserved residues, black letters on a dark gray background; block of similarity, black letters on a light grey background; identical residues, white letters on a black background; The GenBank accession numbers of the sequences used are as follows: Bombyx mori prophenoloxidase activating factor 3 (BmPPAF-3: AAL31707.1), Culex quinquefasciatus serine proteinase (CqSP: XP 001842728.1), Callinectes sapidus prophenoloxidase activating enzyme 3 (CsPAE-3: ABG67960.1), Macrobrachium rosenbergii prophenoloxidase activating enzyme 3 (MrPAE-3: AET34914.1), Fenneropenaeus chinensis serine proteinase (FcSP: AFW98988.1), Litopenaeus vannamei serine proteinase 1 (LvSP1: AFW98995.1), Penaeus monodon serine proteinase 1 (PmSP-1: ACP19562.1), Fenneropenaeus chinensis prophenoloxidase activating enzyme 2 (FcPAE-2: AFW98984.1), Litopenaeus vannamei prophenoloxidase activating enzyme 2 (LvPAE-2: AFW98992.1), Penaeus monodon prophenoloxidase activating enzyme 2 (PmPAE-2: ACP19559.1), Scylla paramamosain serine proteinase (SpSP: CCW43200.1) and Holotrichia diomphalia prophenoloxidase activating factor 3 (HdPPAF-3:BAC15604.1). 


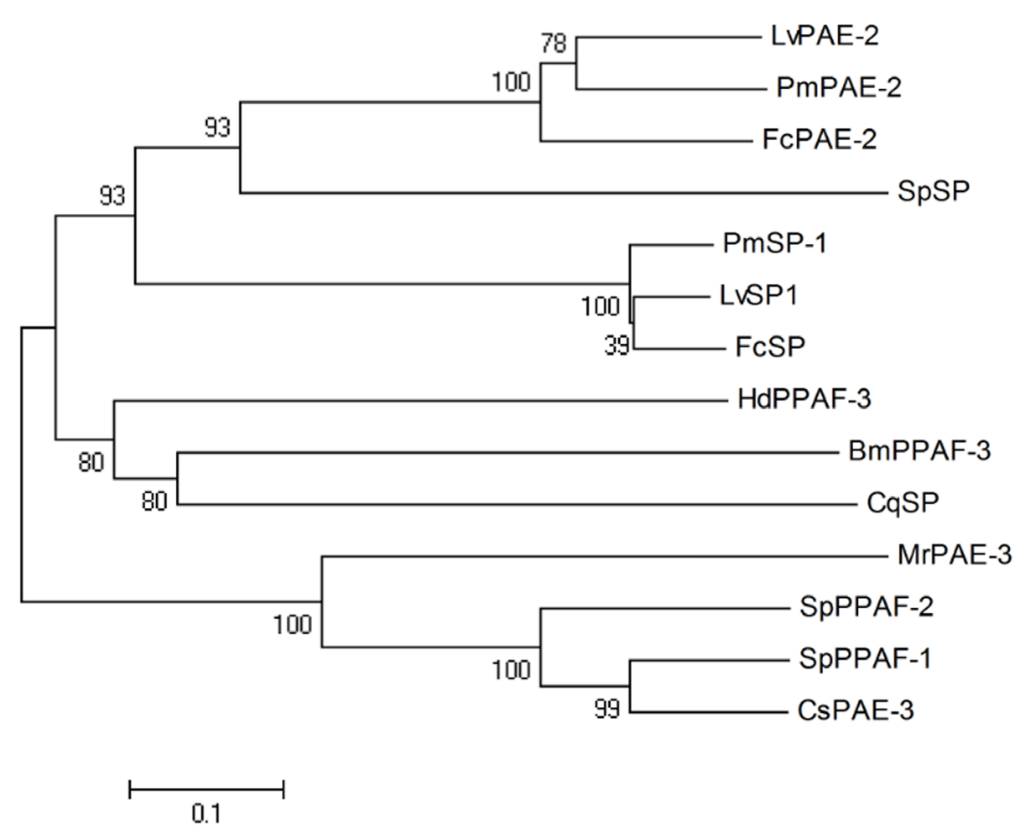

Figure 3. Evolutionary tree of prophenoloxidase activating factor (PPAF). The tree was constructed using the neighbor-joining algorithm in the MEGA 4.1 program based on multiple sequence alignment by Clustal W, and the reliability of the branching was tested using bootstrap re-sampling (1000 pseudo-replicates). The scale bar corresponds to estimated amino acid substitutions per site. The GenBank accession numbers of the sequences used are as follows: Bombyx mori prophenoloxidase activating factor 3 (BmPPAF-3: AAL31707.1), Callinectes sapidus prophenoloxidase activating enzyme 3 (CsPAE-3: ABG67960.1), Culex quinquefasciatus serine proteinase (CqSP: XP_001842728.1), Fenneropenaeus chinensis prophenoloxidase activating enzyme 2 (FcPAE-2: AFW98984.1), Holotrichia diomphalia prophenoloxidase activating factor 3 (HdPPAF-3: BAC15604.1), Litopenaeus vannamei prophenoloxidase activating enzyme 2 (LvPAE-2: AFW98992.1), Macrobrachium rosenbergii prophenoloxidase activating enzyme 3 (MrPAE-3: AET34914.1), Penaeus monodon prophenoloxidase activating enzyme 2 (PmPAE-2: ACP19559.1), Scylla paramamosain serine proteinase (SpSP: CCW43200.1), Litopenaeus vannamei serine proteinase 1 (LvSP1: AFW98995.1), Fenneropenaeus chinensis serine proteinase (FcSP: AFW98988.1), and Penaeus monodon serine proteinase 1 (PmSP-1: ACP19562.1).

\section{Protein structure of $S p P P A F$}

To further study the function of SPPPAF and its secondary structure, a 3-D model was established using the Predic Protein software (https://www.predictprotein.org/) and the SWISS-MODEL long-distance server. The Predic Protein software provided a structural model of both SpPPAF1 and SpPPAF2. The results showed that SPPPAF1 and SpPPAF2 included 7.7 and 5.9\% alpha helix, 29.3 and $31.8 \%$ extended strand, and 63.0 and $62.3 \%$ random coil, respectively, suggesting that the random coil was the major component in $S p P P A F$. The deduced amino-acid sequences of $S p P P A F 1$ and $S p P P A F 2$ were submitted to the SWISS-MODEL server. By searching the Protein Data Bank (PDB) database with this program, a structure with respective 33.74 and $31.9 \%$ identity with the porcine trypsinlike serine proteinase (PDB accession No. 2pkaY) was obtained. The comparative analysis revealed that although there were some differences in the PPAF protein sequences, the spatial structure was highly conserved in various species (Figure 4a and b). 
a

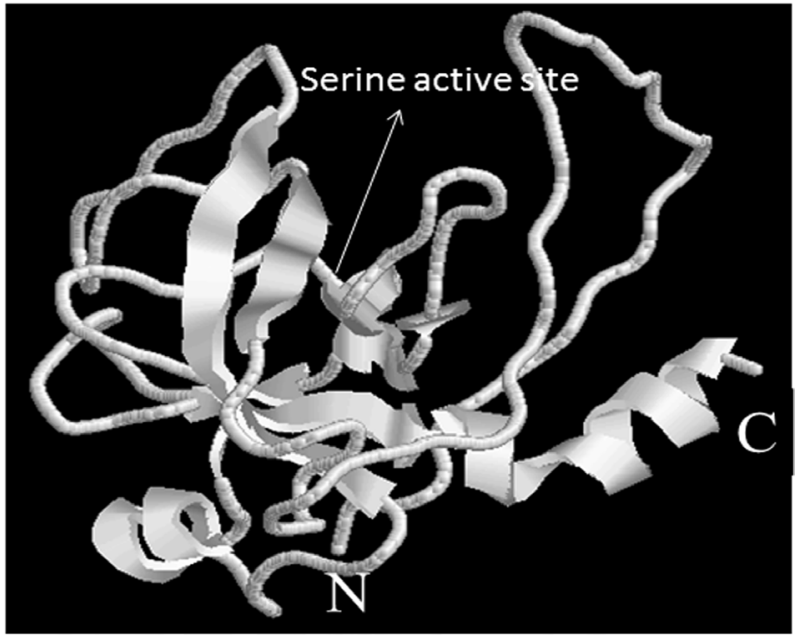

b



Figure 4. Predicted 3-D model of (a) Scylla paramamosain prophenoloxidase activating factor 1 (SpPPAF1) and (b) SPPPAF2, established based on the Swiss Model at the website. "N" indicates the N-terminal residue; "C" indicates the $\mathrm{C}$-terminal residue.

\section{qRT-PCR analysis of SpPPAF in different tissues}

The qRT-PCR investigation was carried out to identify the spatial and temporal expression of SpPPAF in different tissues. As shown in Figure 5a, SpPPAF1 mRNA was highly expressed in the gill, testis, and hemocytes, with lower expression in the ovary, hepatopancreas, muscle, and heart. SPPPAF 2 mRNA was mainly expressed in hemocytes, with lower expression in the other tissues. In addition, the overall expression level of $S p P P A F 1$ was higher than that of SpPPAF2 in the different tissues. 


\section{Temporal expression of SpPPAF mRNA in megalopa stage after bacterial challenge}

Temporal expression of the SPPPAF1 and SpPPAF2 genes in the megalopa stage of $S$. paramamosain after $V$. parahaemolyticus challenge was investigated using qRT-PCR. The results showed that bacterial injection affected the transcript abundance of the SpPPAF gene. As shown in Figure $5 \mathrm{~b}$, there was a slight difference in the expression of $S p P P A F 1$ and $S p P P A F 2$ during the first $6 \mathrm{~h}$ following the challenge. SPPPAF1 mRNA expression declined during the first $3 \mathrm{~h}$, and was then upregulated during the next $9 \mathrm{~h}$, reaching its highest expression $12 \mathrm{~h}$ after the challenge, and then expression was gradually downregulated, reaching its original level $72 \mathrm{~h}$ after the challenge. SPPPAF 2 mRNA expression increased during the first $3 \mathrm{~h}$, then was downregulated during the subsequent $3 \mathrm{~h}$, after which its expression followed the same trend as expression of SPPPAF1, finally reaching its original expression level $72 \mathrm{~h}$ after the challenge.



Figure 5. Relative expression levels of Scylla paramamosain prophenoloxidase activating factor $(S p P P A F)$ in (a) various tissues of adults and (b) in the megalopa stage after Vibrio parahaemolyticus challenge, detected using quantitative real-time PCR. The amount of PPAF mRNA was normalized by the $18 S \mathrm{rRNA}$ transcript level. Data are reported as means $\pm \mathrm{SD}$ of three repeated experiments. The $\mathrm{y}$-axis represents the relative ratio of expression levels of $S p P P A F / 18$ SrRNA mRNA. 


\section{DISCUSSION}

The pro-PO activating system is considered to be an important innate defense mechanism in invertebrate immunity. The proteolytic cleavage of the inactive pro-PO into the active form of PO is a regulated event and is mediated by a cascade of clip-SPs (Gupta et al., 2005; Charoensapsri et al., 2009). Previous studies have demonstrated that several members of clip-SPs are essential in the regulation of the pro-PO activating cascade in insects (Jiang and Kanost, 2000; Barillas-Mury, 2007; Jang et al., 2008). The function of clip-SPs has also been reported to be involved in the pro-PO activating system of some crustaceans (Charoensapsri et al., 2009, 2011; Ma et al., 2014). However, there is little knowledge about the activation and regulation of clip-SPs involved in the $S$. paramamosain pro-PO activation pathway. In this study, we cloned the first two full-length cDNA sequences of PPAF from S. paramamosain.

Based on the predicted amino acid sequences of $S P P P A F 1$ and $S p P P A F 2$, the two proteins contain a highly conserved C-terminal trypsin-like proteinase domain and an $\mathrm{N}$-terminal clip domain, like most of the other known arthropod PPAFs. A previous study reported that the clip domain in PPAF had antibacterial activity in vitro (Wang et al., 2001). In the clip domain of $S p P P A F$, six cysteine residues were identified, which is similar to the clip domain of PPAF from other arthropods, such as P. monodon (Ma et al., 2014) and C. sapidus (Buda and Shafer, 2005). By comparing the canonical clip domains with other SPs, it is predicted that these cysteines will form three pairs of disulfide bonds and thus, are expected to form quite a compact structure. The C-terminal trypsin-like serine proteinase domain of SpPPAF exhibits a characteristic feature of typical serine proteinases, including the conserved His, Asp, and Ser residues, which participate in enzyme catalysis, suggesting that the two proteins are catalytic serine proteinases. However, in the serine proteinase homologue family, the typical catalytic triad (His, Asp, and Ser) found in most serine proteinases is modified to His, Asp, and Gly, as in the case of PmPPAF (Ma et al., 2014). Actually, the clip-SPs are classified as catalytic and non-catalytic groups according to their proteolytic activity. The non-catalytic group members (SPHs) do not exhibit any proteolytic activity owing to replacement of the serine residue at the active site by glycine (Charoensapsri et al., 2009). A previous study has reported that the SPHs lack enzyme activity due to a modified proteinase domain, which has been suggested to mediate protein-protein interaction or to act as an antagonist of serine proteinase to regulate and control enzyme activity (Ma et al., 2014).

mRNA expression level is a substantial element that affects the synthesis of PPAF. In this study, we identified the tissue distribution of $S p P P A F$ using qRT-PCR and found that $S p P P A F$ was detectable in nearly all tissues including the gill, testis, hemocytes, ovary, hepatopancreas, muscle, and heart. $S p P P A F 1$ mRNA was highly expressed in the gill, testis, and hemocytes, while SPPPAF2 mRNA was mainly expressed in hemocytes. In addition, the overall expression level of $S P P P A F 1$ was higher than that of $S P P P A F 2$ in the different tissues. It was reported that the PmPPAF transcript in P. monodon was detectable in the hypodermis, hemocytes, gill, swimming leg, brain, and eyestalk but not in the hepatopancreas, heart, muscle, thoracic ganglion, and ventral nerve cord (Ma et al., 2014). In addition, PmPPAF1 and PMPPAF2 were found to be expressed only in hemocytes but not in other tissues (Charoensapsri et al., 2009, 2011), which is similar to the expression profile of PPAF mRNA in $P$. leniusculus (Wang et al., 2001). These results suggest that the expression level of the PPAF gene is different in diverse species, probably because PPAF acts in different ways in different tissues of these species. However, the gills and hemocytes may be the two main sites for synthetizing $P P A F$ genes and they may play an important role in crustacean defense against 
bacterial infection. In addition, the extensive expression of the $S p P P A F$ gene in other tissues suggests that $S p P P A F$ may have a broader function, such as involvement in the melanization process (Ma et al., 2014).

Knowing that the megalopa stage is the most important stage in crab cultivation, we chose the megalopa as the subject for investigation in this study, and observed a time-dependent pattern of SpPPAF1 and SpPPAF 2 mRNA expression after V. parahaemolyticus challenge. Many researchers have reported that the expression level of the PPAF gene varies greatly in different species after bacterial challenge. In Manduca sexta, three PAP transcript expression levels were induced to a high level by bacterial injection (Jiang et al., 1998, 2003a,b). In $P$. monodon, the transcription of PMPPAE 1 was reduced between 3 and $24 \mathrm{~h}$ after Vibrio harveyi challenge, and then increased significantly at $48 \mathrm{~h}$ after infection. In the current study, a slight difference in the expression levels of $S p P P A F 1$ and $S p P P A F 2$ were observed during the first 6 $\mathrm{h}$ after the challenge. SPPPAF1 mRNA expression declined during the first $3 \mathrm{~h}$ and was then upregulated during the next $3 \mathrm{~h}$, while SPPPAF 2 mRNA expression was upregulated during the first $3 \mathrm{~h}$ and then downregulated during the next $3 \mathrm{~h}$. However, the expression levels of $S P P P A F 1$ and SpPPAF2 shared the same trend as follows: both reached peak expression $12 \mathrm{~h}$ after the challenge, and then expression was gradually downregulated, reaching the original level $72 \mathrm{~h}$ after the challenge. Therefore, we assume that the expression of both SPPPAF 1 and $S P P P A F 2$ is related to the bacterial challenge but the expression patterns differ, although this assumption needs to be confirmed with additional research.

It has been reported that more than a single gene encodes PPAF in some species. For example, three PPAFs have been isolated from the insect $M$. sexta and their cloned cDNAs can directly activate pro-PO (Yu et al., 2003). In Drosophila, five putative PPAE genes have been studied (Gregorio et al., 2001). In P. monodon, three PPAFs have been obtained (Charoensapsri et al., 2009, 2011; Ma et al., 2014). In this study, we cloned two PPAF cDNAs from S. paramamosain (SPPPAF1 and SPPPAF2). As a multifunctional molecule, PPAF has a number of different functions, including controlling and regulating molting. The most recognized function is that of working as a zymogen to participate in the activation of the pro-PO system. However, Wang et al. (2001)'s group demonstrated that one active serine protease is sufficient for the activation of fresh water crayfish P. leniusculus pro-PO. While, Ashida and Yoshida (1988) suggested that at least two serine proteases were required for the activation of the pro-PO system in the hemolymph of $B$. mori, one of which activated pro-PO through limited proteolysis. In addition, in Hyalophora cecropia, one serine protease and an additional unknown factor could convert pro-PO to an active enzyme (Anderson et al., 1989). As previously reported by Wang et al. (2001), the two SpPPAF in our study perhaps play two different roles in crab innate immunity. One may work as a key enzyme in the pro-PO activating system and the other may produce an antibacterial fragment that directly attacks the bacteria at localized places, such as in melanized nodules. Although the relationship between SpPPAF1 and $S p P P A F 2$, and how these two molecules activate the pro-PO activation pathway remain unclear, we have obtained pro-PO and several other factors of SPs/SPHs from the pro-PO activation pathway in S. paramamosain (unpublished data). Further investigation should focus on the mechanism underlying the regulatory role of PPAF in the immune system.

\section{Conflicts of interest}

The authors declare no conflict of interest. 


\section{ACKNOWLEDGMENTS}

Research supported by the National Natural Science Foundation of China (\#31101890, \#31310103032), the Science and Technology Commission of Shanghai Municipality (\#10JC1418600), and the Basic Research Fund for State-level Nonprofit Research Institutes of ESCFRI, CAFS (\#2014Z01-2, \#2012T05).

\section{REFERENCES}

Amparyup P, Promrungreang K, Charoensapsri W, Sutthangkul J, et al. (2013). A serine proteinase PmClipSP2 contributes to prophenoloxidase system and plays a protective role in shrimp defense by scavenging lipopolysaccharide. Dev. Comp. Immunol. 41: 597-607.

Anderson K, Sun SC, Boman HG and Steiner H (1989). Purification of the prophenoloxidase from Hyalophora cecropia and four proteins involved in its activation. Insect Biochem. 19: 629-637.

Ashida M and Yoshida H (1988). Limited proteolysis of prophenoloxidase during activation by microbial products in insect plasma and effect of phenoloxidase on electrophoretic mobilities of plasma proteins. Insect Biochem. 18: 11-19.

Barillas-Mury C (2007).CLIP proteases and Plasmodium melanization in Anopheles gambiae. Trends Parasitol. 23: 297-299.

Buda ES and Shafer TH (2005). Expression of a serine proteinase homologprophenoloxidase-activating factor from the blue crab, Callinectes sapidus. Comp. Biochem. Physiol. B-Biochem. Mol. Biol. 140: 521-531.

Cerenius L and Söderhäll K (2004). The prophenoloxidase-activating system in invertebrates. Immunol. Rev. 198: 116-126.

Charoensapsri W, Amparyup P, Hirono I, Aoki T, et al. (2009). Gene silencing of a prophenoloxidase activating enzyme in the shrimp, Penaeus monodon, increases susceptibility to Vibrio harveyi infection. Dev. Comp. Immunol. 33: 811-820.

Charoensapsri W, Amparyup P, Hirono I, Aoki T, et al. (2011). PmPPAE2, a new class of crustacean prophenoloxidase (proPO)-activating enzyme and its role in PO activation. Dev. Comp. Immunol. 35: 115-124.

Gupta S, Wang Y and Jiang H (2005). Manduca sexta prophenoloxidase (proPO) activation requires proPO-activating proteinase (PAP) and serine proteinase homologs (SPHs) simultaneously. Insect Biochem. Mol. Biol. 35: 241-248.

Gregorio ED, Spellman PT, Rubin GM and Lemaitre B (2001). Genome-wide analysis of the Drosophila immune response by using oligonucleotide microarrays. Proc. Natl. Acad. Sci. U. S. A. 98: 12590-12595.

Jang IH, Nam HJ and Lee WJ (2008). CLIP-domain serine proteases in Drosophila innate immunity. BMB Rep. 41: 102-107.

Jang IK, Pang Z, Yu J, Kim SK, et al. (2011). Selectively enhanced expression of prophenoloxidase activating enzyme 1 (PPAE1) at a bacteria clearance site in the white shrimp, Litopenaeus vannamei. BMC Immunol. 12: 70.

Jiang HB and Kanost MR (2000). The clip-domain family of serine proteinases in arthropods. Insect Biochem. Mol. Biol. 30: $95-105$.

Jiang HB, Wang Y and Kanost MR (1998). Pro-phenol oxidase activating proteinase from an insect, Manduca sexta: a bacteria-inducible protein similar to Drosophilaeaster. Proc. Natl. Acad. Sci. U. S. A. 95: 12220-12225.

Jiang HB, Wang Y, Yu XQ, Zhu Y, et al. (2003a). Prophenoloxidase-activating proteinase-3 (PAP-3) from Manduca sexta hemolymph: a clip-domain serine proteinase regulated by serpin-1 J and serine proteinase homologs. Insect Biochem. Mol. Biol. 33: 1049-1060.

Jiang HB, Wang Y, Yu XQ and Kanost MR (2003b). Prophenoloxidase-activating Proteinase-2 from Hemolymph of Manduca sexta: a bacteria-inducible serine proteinase containing two clip domains. J. Biol. Chem. 278: 3552-3561.

Jiménez-Vega F, Vargas-Albores F and Soderhall K (2005). Characterisation of a serine proteinase from Penaeus vannamei haemocytes. Fish Shellfish Immunol. 18: 101-108.

Kanost MR, Jiang H, Wang Y, Yu XQ, et al. (2001). Hemolymph proteinases in immune responses of Manduca sexta. Adv. Exp. Med. Biol. 484: 319-328.

Kawabata S, Tokunaga F, Kugi Y, Motoyama S, et al. (1996). Limulus factor D, a 43-kDa protein isolated from horseshoe crab hemocytes, is a serine protease homologue with antimicrobial activity. FEBS Lett. 398: 146-150.

Kwon TH, Kim MS, Choi HW, Joo CH, et al. (2000). A masquerade-like serine proteinase homologue is necessary for phenoloxidase activity in the coleopteran insect, Holotrichia diomphalia larvae. Eur. J. Biochem. 267: 6188-6196.

Ma TH, Benzie JA, He JG, Sun CB, et al. (2014). PmPPAF is a pro-phenoloxidase activating factor involved in innate immunity response of the shrimp Penaeus monodon. Dev. Comp. Immunol. 44: 163-172.

Satoh D, Horii A, Ochiai M and Ashida M (1999). Prophenoloxidase-activating enzyme of the silkworm, Bombyx mori. Purification, characterization, and cDNA cloning. J. Biol. Chem. 274: 7441-7453.

Söderhäll K and Cerenius L (1998). Role of the prophenoloxidase-activating system in invertebrate immunity. Curr. Opin. Immunol. 10: 23-28. 
Söderhäll K, Cerenius L and Johansson MW (1994). The prophenoloxidase activating system and its role in invertebrate defence. Ann. NY Acad. Sci. 712: 155-161.

Tamura K, Peterson D and Peterson N (2011). MEGA5: molecular evolutionary genetics analysis using maximum likelihood, evolutionary distance, and maximum parsimony methods. Mol. Biol Evol. 28: 2731-2739.

Wang R, Lee SY, Cerenius L and Söderhäll K (2001). Properties of the prophenoloxidase activating enzyme of the freshwater crayfish, Pacifastacus leniusculus. Eur. J. Biochem. 268: 895-902.

Wang Y, Lu Z and Jiang H (2014). Manduca sexta proprophenoloxidase activating proteinase-3 (PAP3) stimulates melanization by activating proPAP3, proSPHs, and proPOs. Insect Biochem. Mol. Biol. 50: 82-91.

Yu XQ, Jiang H, Wang Y and Kanost MR (2003). Nonproteolytic serine proteinase homologs are involved in prophenoloxidase activation in the tobacco hornworm, Manduca sexta. Insect Biochem. Mol. Biol. 33: 197-208.

Zhang D, Jiang K, Zhang F, Ma C, et al. (2011). Isolation and characterization of a ferritin cDNA from the mud crab Scylla paramamosain. J. Crustac. Biol. 31:345-351. 